\title{
Deacetylation of XBP1s by sirtuin 6 confers resistance to ER stress-induced hepatic steatosis
}

\author{
In Hyuk Bang ${ }^{1}$, Oh Kwang Kwon², Lihua Hao ${ }^{1}$, Dami Park', Myung-Ja Chung ${ }^{3}$, Byung-Chul Oh${ }^{4}$, Sangkyu Lee², \\ Eun Ju Bae ${ }^{5}$ and Byung-Hyun Park
}

\begin{abstract}
The active spliced form of X-box-binding protein 1 (XBP1s) is a key modulator of ER stress, but the functional role of its post-translational modification remains unclear. Here, we demonstrate that XBP1s is a deacetylation target of Sirt6 and that its deacetylation protects against ER stress-induced hepatic steatosis. Specifically, the abundance of acetylated XBP1s and concordant hepatic steatosis were increased in hepatocyte-specific Sirt6 knockout and obese mice but were decreased by genetic overexpression and pharmacological activation of Sirt6. Mechanistically, we identified that Sirt6 deacetylated a transactivation domain of XBP1s at Lys 257 and Lys297 and promoted XBP1s protein degradation through the ubiquitin-proteasome system. Overexpression of XBP1s, but not its deacetylation mutant 2KR (K257/297R), in mice increased lipid accumulation in the liver. Importantly, in liver tissues obtained from patients with nonalcoholic fatty liver disease (NAFLD), the extent of XBP1s acetylation correlated positively with the NAFLD activity score but negatively with the Sirt6 level. Collectively, we present direct evidence supporting the importance of XBP1 acetylation in ER stress-induced hepatic steatosis.
\end{abstract}

\section{Introduction}

Nonalcoholic fatty liver disease (NAFLD) is a disorder characterized by hepatic fat accumulation (steatosis) that can progress to nonalcoholic steatohepatitis (NASH) ${ }^{1}$. During chronic high-calorie diet feeding, an increased demand for protein synthesis leads to the disruption of endoplasmic reticulum (ER) homeostasis, which is termed ER stress ${ }^{2}$. ER stress, in turn, induces the unfolded protein response (UPR) that directs unfolded or misfolded proteins to molecular chaperones to be refolded or degraded. In mammalian cells, the UPR is initiated by three ER stress sensor proteins: inositol-requiring enzyme $1 \alpha$ (IRE1 $\alpha$ ), protein kinase-like ER kinase (PERK), and activating transcription factor 6 (ATF6) $)^{3}$. Upon exposure to ER stressors, IRE1 $\alpha$ undergoes dimerization and

\footnotetext{
Correspondence: Eun Ju Bae (ejbae7@jbnu.ac.kr) or Byung-Hyun Park (bhpark@jbnu.ac.kr)

'Department of Biochemistry, Chonbuk National University Medical School, Jeonju, Jeonbuk 54896, Republic of Korea

${ }^{2}$ College of Pharmacy, Kyungpook National University, Daegu 41566, Republic of Korea

Full list of author information is available at the end of the article.
}

transautophosphorylation, which activates its endoribonuclease activity and cleaves XBP1 mRNA, leading to the translation of a spliced form of XBP1 (XBP1s) ${ }^{4}$. As a transcription factor, XBP1s upregulates the expression of specific genes involved in ER biogenesis and protein secretion, as well as molecular chaperones ${ }^{5}$.

Although ER stress has been implicated in a variety of liver diseases, including NAFLD ${ }^{5-7}$, how ER stress causes lipid accumulation in the liver remains largely unknown. Several mechanisms through which UPR molecules modulate ER stress-induced steatosis have been proposed. For example, a recent report showed that VLDL receptor upregulation by ER stress and subsequent lipid uptake mediate hepatic steatosis ${ }^{8}$. On the other hand, IRE1 $\alpha$ has been shown to prevent ER stress-induced fatty liver development, as IRE1 $\alpha$ knockout (KO) increased steatosis $^{9,10}$. Notably, hepatocyte-specific deletion of XBP1s protected mice from hepatic steatosis and insulin resistance ${ }^{11,12}$, identifying XBP1s as a transcriptional activator of de novo lipogenic genes. Similarly, high-fat diet (HFD) feeding induces XBP1s in the liver, which mediates

\section{(c) The Author(s) 2019}

(c) (i) Open Access This article is licensed under a Creative Commons Attribution 4.0 International License, which permits use, sharing, adaptation, distribution and reproduction c. in any medium or format, as long as you give appropriate credit to the original author(s) and the source, provide a link to the Creative Commons license, and indicate if changes were made. The images or other third party material in this article are included in the article's Creative Commons license, unless indicated otherwise in a credit line to the material. If material is not included in the article's Creative Commons license and your intended use is not permitted by statutory regulation or exceeds the permitted use, you will need to obtain permission directly from the copyright holder. To view a copy of this license, visit http://creativecommons.org/licenses/by/4.0/. 
hepatic insulin resistance ${ }^{13}$. Thus, the downregulation of $\mathrm{XBP} 1 \mathrm{~s}$ seems to have potential as an approach for inhibiting ER stress-induced hepatic steatosis.

Sirtuins (from Sirt1 to Sirt7) have been implicated in NAFLD development under ER stress. Activation of Sirt1 by resveratrol prevents the development of NAFLD in HFD-fed mice through the suppression of ER stress ${ }^{14}$, suggesting that Sirt1 is a negative regulator of ER stress. Recent reports have shown that Sirt1 deacetylates XBP1s and inhibits its transcriptional activity, whereas acetyltransferase p300 increases the acetylation and protein stability of XBP1s ${ }^{15}$. Similarly, Sirt7 functions at the chromatin level to suppress ER stress and prevents NAFLD development ${ }^{16}$. Sirt6, a nuclear form of sirtuin, deacetylates the lysine residues of histone (H3K9, H3K18, and $\mathrm{H} 3 \mathrm{~K} 56)^{17-19}$ and non-histone substrates ${ }^{20-23}$. Our group and others have found that mice lacking Sirt6 in hepatocytes develop NAFLD ${ }^{24,25}$ and that Sirt6 overexpression suppresses oxidative stress and inflammation in the liver under a high-calorie diet ${ }^{24}$. We also observed a decrease in Sirt6 protein levels in the liver of obese mice and NAFLD patients ${ }^{24}$; however, the role of Sirt6 in the ER stress response has not been studied.

In this study, we aimed to determine whether deletion, overexpression, or pharmacological activation of Sirt6 alters ER stress-induced hepatic steatosis and the associated pathology and to investigate the molecular mechanism of Sirt6 regulation of the UPR elicited by ER stress.

\section{Materials and methods \\ Animals}

Hepatocyte-specific Sirt6 KO mice (Sirt6 ${ }^{\text {flox/flox }}$;albumin-Cre) were generated, as described previously ${ }^{24}$. Male Sirt6 KO mice and wild-type (albumin-Cre) littermates older than 6 weeks of age were fed a normal chow diet (NCD), a $60 \%$ HFD, or a $60 \%$ fat and 30\% fructose diet (HFHF) for 10 weeks ad libitum. For the tunicamycin (Tm)-induced ER stress animal experiment, mice at 8-9 weeks of age were administered DMSO or Tm (Sigma-Aldrich, St. Louis, MO, USA) intraperitoneally at a dose of $1 \mathrm{mg} / \mathrm{kg}$ body weight. Sirt6 adenoviruses were injected intravenously via the tail vein two days before $\mathrm{Tm}$ treatment. All experimental procedures were approved by the Institutional Animal Care and Use Committee of Chonbuk National University (permit number: CBNU-2015-088).

\section{Human tissues}

Human liver tissues were obtained from Chonbuk National University Hospital Biobank (CNUHB), with informed consent from the patients, and the study was approved by the Institutional Review Board of Chonbuk National University (permit number: T14-18) ${ }^{24}$. To assess morphological changes in the liver, we used the NAFLD activity score (NAS) ${ }^{26}$.

\section{Cell culture and transient transfection}

For isolation of primary hepatocytes, mouse livers were perfused with collagenase type IV (Sigma-Aldrich), and hepatocytes were prepared as described previously ${ }^{27}$. The human hepatoma cell line HepG2 and human embryonic kidney cell line HEK293T were obtained from the American Type Culture Collection (Manassas, VA, USA). Exogenous proteins were expressed by transfecting HEK293T cells with $1 \mu \mathrm{g}$ of XBP1s, Sirt6, Sirt6-H133Y (mSirt6), and p300 using Lipofectamine 3000 (Invitrogen, Carlsbad, CA, USA). For the XBP1s reporter gene assay, $2 \mu \mathrm{g}$ of the ER stress response element (ERSE) luciferase promoter (Qiagen, Hilden, Germany) was used.

\section{Antibodies}

Antibodies against the following proteins were used: Sirt6, p-Akt, Akt, Bax, cleaved caspase-3, Ac-K, AcH3K56, ATF4 (Cell Signaling, Beverly, MA, USA), GRP78, p-PERK, p-eIF2 $\alpha$, XBP1s, ubiquitin, ATF6, Sirt1 (Santa Cruz Biochemicals, Dallas, TX, USA), p-IRE1 $\alpha$ (Abcam, Cambridge, UK), CHOP, lamin B, GAPDH (Bioworld Technology, St. Louis Park, MN, USA), Ac-H3K9, and $\beta$-actin (Sigma-Aldrich).

\section{Liquid chromatography (LC)-mass spectrometry (MS/MS) analysis}

Immunoprecipitated proteins were visualized by staining SDS gels with colloidal Coomassie blue. After protein bands were excised from the gels, the proteins were subjected to in-gel digestion with trypsin. The tryptic peptides were analyzed by a high-resolution mass spectrometer (LTQ-Orbitrap Velos, Thermo Fisher Scientific, Waltham, MA, USA) coupled with nanoflow liquid chromatography (nano-LC) for acetylation, as described previously $^{28}$.

\section{Site-directed mutagenesis}

Acetylation mutants of XBP1s were generated using a site-directed mutagenesis kit (GeneAll, Seoul, Korea) by converting each lysine residue (K65, K146, K257, and K297) to arginine (codon change from AAG or AAA to AGG). Mutations were confirmed by DNA sequencing performed by Cosmogenetech (Seoul, Korea).

\section{Statistical analysis}

The data are expressed as the mean \pm standard error of the mean (SEM). Statistical comparisons were made using one-way analysis of variance followed by Fisher's post hoc analysis. The significance of differences between two groups was determined using Student's unpaired t-test. A $p$ value of less than 0.05 was considered significant. 


\section{Additional methods}

Detailed methods and primer sequences are provided in the Supplementary Information.

\section{Results}

Severe hepatic steatosis in Sirt6 KO mice is associated with upregulation of the UPR

We and others have previously reported a reduction in the expression of Sirt6 in fatty livers ${ }^{24,25}$. To determine whether Sirt6 expression is altered by ER stress, we determined Sirt6 expression after treatment with ER stress inducers in hepatocytes. A decrease in the level of Sirt6 protein was observed after treatment with Tm, thapsigargin, and dithiothreitol, likely due to transcriptional suppression, as supported by the reduction in Sirt6 mRNA level after Tm treatment (Fig. S1a, b). Sirt6 downregulation by $\mathrm{Tm}$ was reversed by co-treatment with the chemical chaperone 4-phenylbutyrate (Fig. S1c). When mice were injected with Tm, Sirt6 expression in the liver decreased at $24 \mathrm{~h}$ and was restored at $48 \mathrm{~h}$ (Fig. S1d). At the same time, and consistent with previous reports ${ }^{8}$, hepatic triglyceride (TG) levels were highly increased, whereas the plasma TG level gradually decreased (Fig. S1e), and these changes were accompanied by increased expression of UPR signaling molecules, including XBP1s, p-PERK, p-eIF2 $\alpha$ and ATF6 (p50) (Fig. S1f). These data imply that ER stress-induced hepatic steatosis may be linked to Sirt6 downregulation.

To investigate the direct role of Sirt6 during the development of ER stress-induced hepatic steatosis, we compared the response of WT and KO mice to a Tm challenge. Sirt6 deficiency led to a robust increase in hepatic fat accumulation upon $\mathrm{Tm}$ administration as assessed by hepatic TG levels (Fig. 1a) and immunohistochemical staining for lipid droplets (Fig. 1b). Basal levels of plasma TG were significantly increased in $\mathrm{KO}$ mice, whereas Tm treatment suppressed plasma TG levels in WT and KO mice (Fig. 1a).

Because the hepatic UPR is also linked with oxidative stress, apoptosis, and insulin resistance ${ }^{2}$, we next examined the effects of Sirt6 deficiency on these parameters. Tm treatment significantly decreased superoxide dismutase activity and glutathione levels in the livers of $\mathrm{KO}$ mice compared with those in WT mice (Fig. 1c). Tm-induced apoptosis and insulin resistance, which were assessed by western blotting for pro-apoptotic molecules or Akt phosphorylation and TUNEL staining, were more evident in Sirt6 KO livers than in WT livers (Figs. 1d-f, S2).

\section{XBP1s activation is a major determinant of the increase in the Tm-induced UPR in Sirt6 KO mice}

To identify the UPR transducers responsible for hepatic steatosis in Sirt6 KO mice, we carefully investigated the activation status of three transducers of ER stress signaling. Although Tm-stimulated phosphorylation of IRE1 $\alpha$ was not different between genotypes, expression levels of XBP1s and its target genes (CHOP, EDEM, and ERDJ4) were greatly increased in Sirt6 KO mice (Figs. 2a, c, S3). Phosphorylation levels of PERK, its downstream molecule p-eIF2 $\alpha$ and GRP78 were also higher in KO mice than in WT mice, while ATF6 (p50) expression was not changed (Figs. 2a, S3). Because XBP1s expression is largely regulated by XBP1 mRNA splicing, we next measured XBP1 mRNA splicing by RT-PCR. As shown in Fig. 2b, Tm-induced XBP1 mRNA splicing was not changed between genotypes, which was in line with the findings of no changes in IRE1 $\alpha$ phosphorylation in $\mathrm{KO}$ mice (Figs. 2a, S3). The Sirt6 deficiency-induced increase in XBP1s levels upon Tm stimulation with no difference in XBP1 mRNA splicing was further confirmed in primary hepatocytes (Fig. 2d).

\section{Sirt6 overexpression alleviates steatosis, apoptosis, and insulin resistance in the liver}

Next, we aimed to assess whether Sirt6 overexpression protects the liver from ER stress. To this end, C57BL/6 mice were injected with AdLacZ, AdSirt6, or AdmSirt6, followed by $\mathrm{Tm}$ exposure for $24 \mathrm{~h}$. Tm-induced hepatic TG accumulation and lower plasma TG levels were completely abolished in mice injected with AdSirt6 but not AdmSirt6 (Fig. 3a). Among the three pathways of UPR, XBP1s- and PERK-dependent pathways were significantly attenuated by hepatic overexpression of Sirt6 (Figs. 3b, S4a). The ERSE reporter assay results indicated that $\mathrm{Tm}$-induced transactivation of XBP1s was significantly diminished by overexpression of Sirt6 but not mSirt6 (Fig. 3c). In agreement with this finding, the XBP1s targets CHOP and ERDJ4 were also significantly suppressed by Sirt6 overexpression (Fig. 3d), confirming the downregulation of the XBP1s signaling pathway by Sirt6. Because Tm-induced hepatocyte apoptosis was increased in Sirt6 KO mice (Fig. 1d, e), we next investigated whether Sirt6 overexpression protects against apoptotic cell death. Western blotting and TUNEL staining demonstrated that apoptosis was almost completely blocked by Sirt6 overexpression (Figs. 3e, S4b, S5). Finally, Tm treatment of HepG2 cells reduced insulin-stimulated Akt phosphorylation, whereas Sirt6 overexpression fully restored hepatic insulin sensitivity (Figs. 3f, S4c).

\section{Sirt6 decreases the stability of the XBP1s protein}

We next investigated the molecular mechanism of XBP1s reduction by Sirt6, with a focus on its deacetylase activity. When HepG2 cells transduced with control or Sirt6 adenovirus were treated with $\mathrm{Tm}$, the expression and acetylation of XBP1s were increased in the control group but not in Sirt6-overexpressing cells, implying that Sirt6 deacetylates XBP1s (Fig. 4a). Because the acetylation 


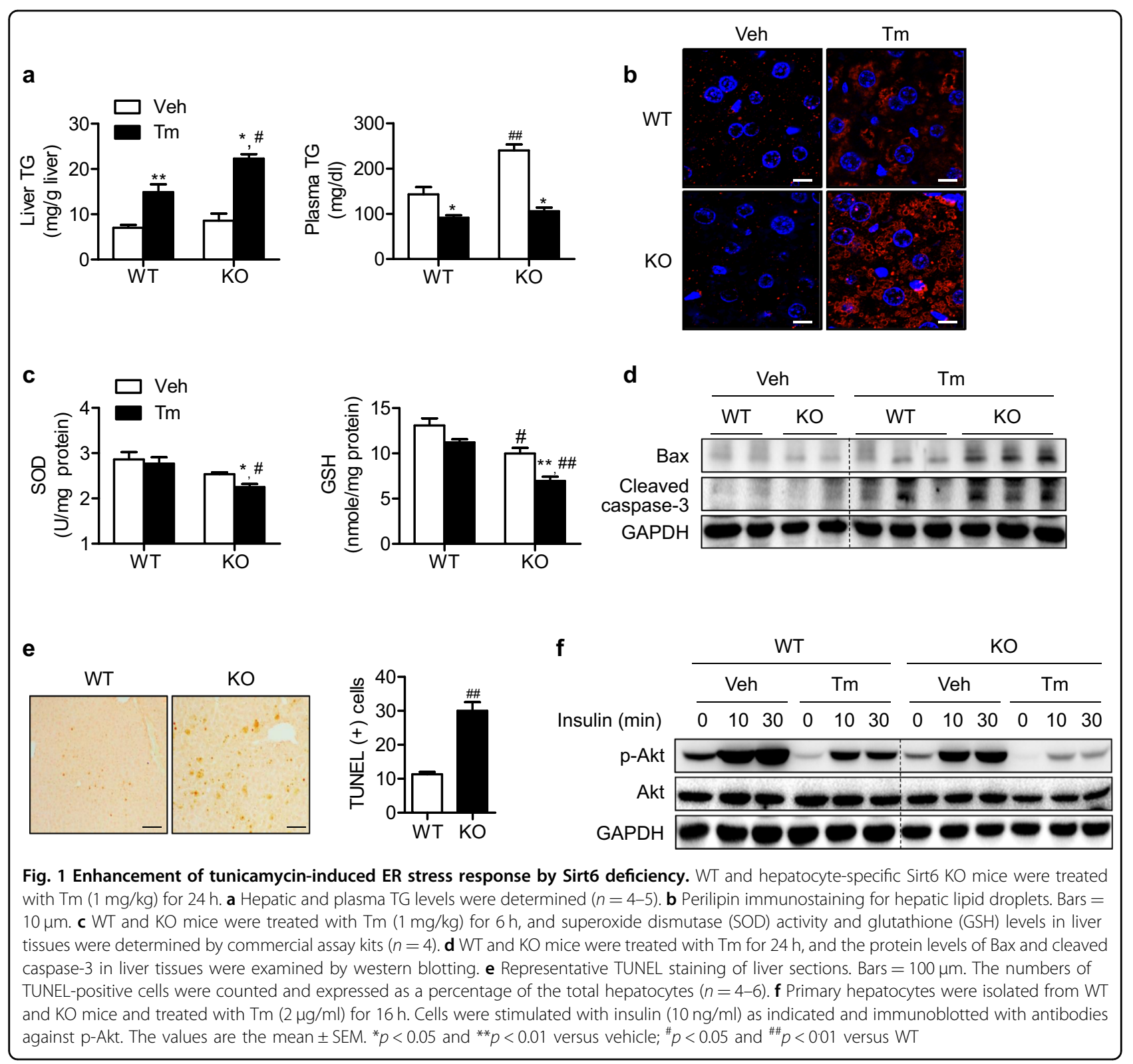

status of XBP1s has been reported to affect its stability ${ }^{15}$, we examined XBP1s stability in HEK293T cells transfected with constructs carrying p300 or XBP1s with or without Sirt6 and then chased the level of XBP1s after treatment with the protein synthesis inhibitor cycloheximide (CHX). CHX treatment gradually decreased the level of XBP1s with a faster rate of disappearance in cells overexpressing Sirt6 (Fig. $4 b)$, indicating that Sirt6 reduces the stability of the XBP1s protein. Indeed, ectopic expression of Sirt6 dosedependently decreased the total protein level of XBP1s in HEK293T cells co-expressing p300 and XBP1s, but deacetylase-inactive mSirt6 had no effect (Fig. 4c). Additional results showed that Sirt6-mediated XBP1s degradation was abolished in the presence of MG132, a proteasome inhibitor (Fig. 4d), indicating that Sirt6 induces proteasomedependent degradation of XBP1s. Accordingly, Sirt6 overexpression increased the ubiquitination of XBP1s (Fig. 4e). Lastly, we repeated the CHX chase assay in primary hepatocytes isolated from WT or Sirt6 KO mice, and the results clearly indicated that Sirt6 deficiency increased the stability of the XBP1s protein (Fig. 4f). These findings suggest that Sirt6, which is reliant on its deacetylase enzymatic activity, causes the proteasomal degradation of XBP1s.

\section{Sirt6 deacetylates XBP1s at Lys65, Lys146, Lys257, and Lys297}

Our finding that XBP1s acetylation was reduced in cells overexpressing Sirt6 was confirmed by additional 


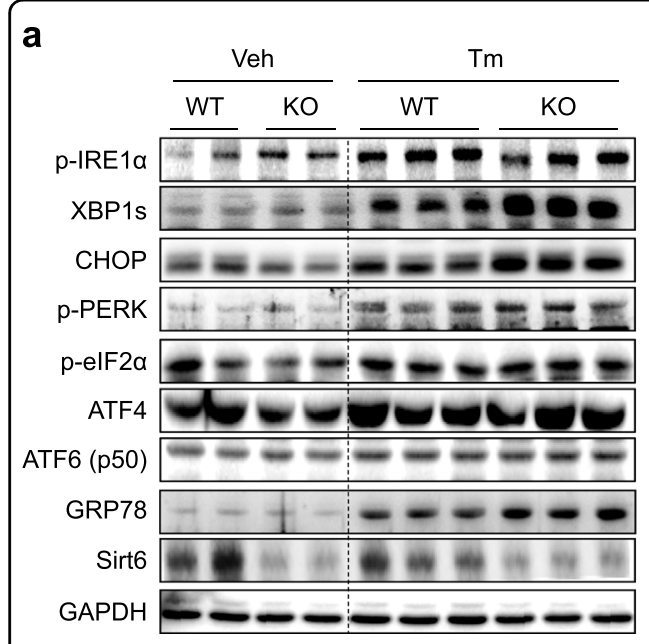

b

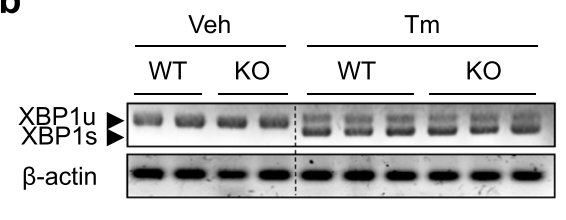

C

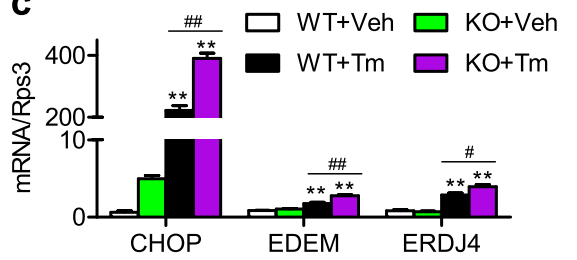

d
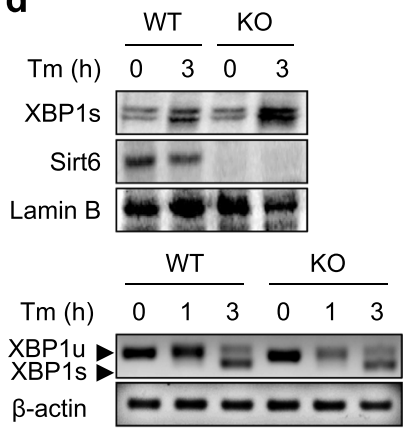

Fig. 2 Activation of the XBP1s pathway by Sirt6 deficiency. WT and KO mice were treated with $T \mathrm{~m}(1 \mathrm{mg} / \mathrm{kg})$ for $24 \mathrm{~h}$. a ER stress markers in the livers were examined by western blotting. b Spliced and unspliced XBP1 mRNA levels were detected using RT-PCR. c The mRNA levels of CHOP, EDEM, and ERDJ4 were analyzed by real-time RT-PCR $(n=4-6)$. $\mathbf{d}$ Primary hepatocytes isolated from WT and KO mice were treated with Tm $(2 \mu \mathrm{g} / \mathrm{ml})$, and the protein levels of XBP1s in the nuclear fraction and XBP1 mRNA splicing were determined by western blotting and RT-PCR, respectively. The values are the mean \pm SEM. ${ }^{* *} p<0.01$ versus vehicle; ${ }^{\#} p<0.05$ and ${ }^{\# \#} p<0.01$ versus $\mathrm{WT}$

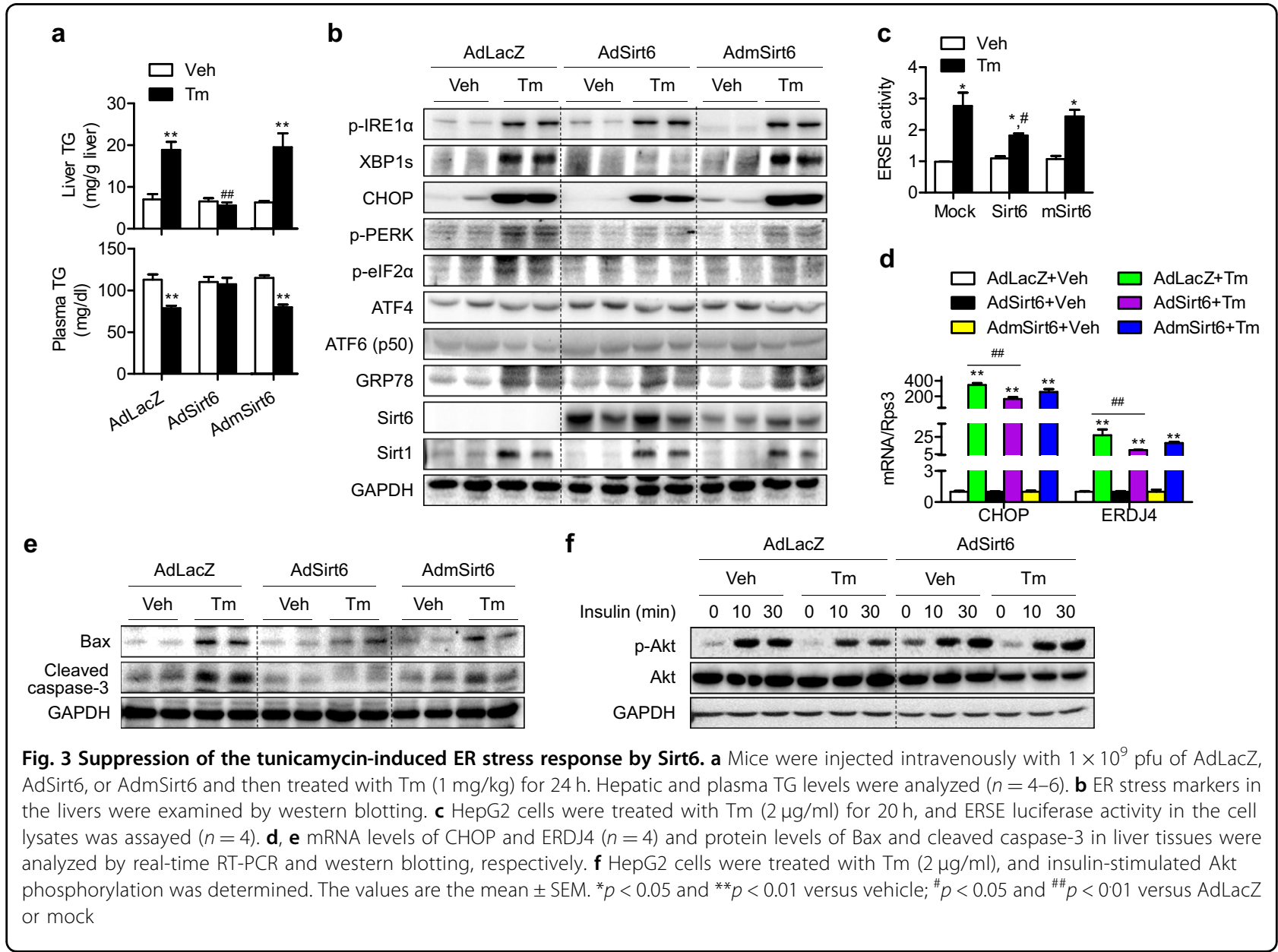




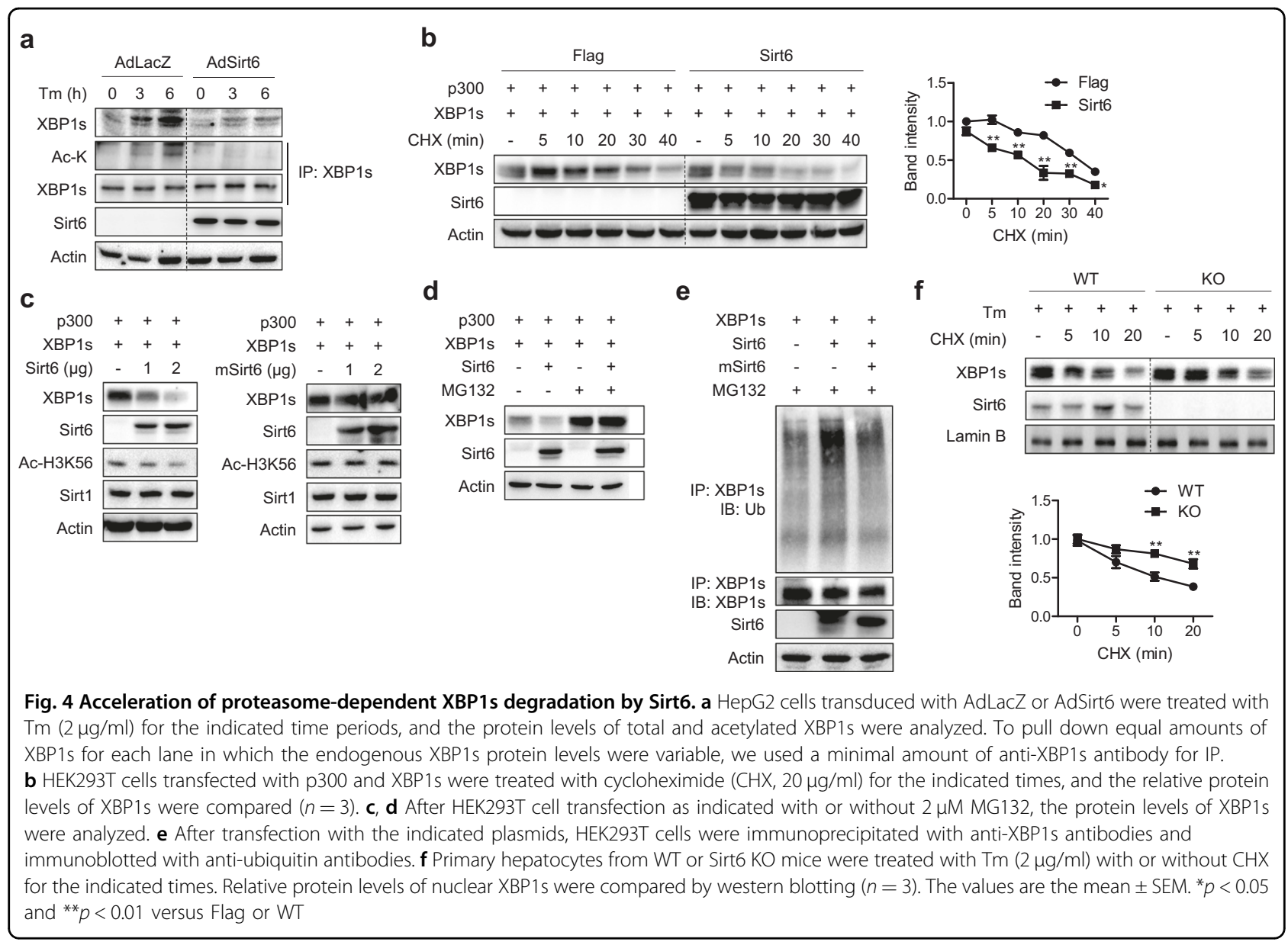

experiments. We co-expressed XBP1s and p300 with either Sirt6 or mSirt6 in HEK293T cells and measured the acetylation level of XBP1s. When the immunoprecipitates of XBP1s were immunoblotted with an anti-acetyl lysine (AcK) antibody, robust acetylation of XBP1s was detected in control cells, but this acetylation was decreased by Sirt6, and catalytically inactive mSirt6 had no effect (Fig. 5a). To further validate these results, we compared the acetylation of XBP1s in primary hepatocytes isolated from WT and Sirt6 KO mice. The results showed an increase in XBP1s acetylation in KO hepatocytes (Fig. 5b). Together, these results suggest that XBP1s is a direct deacetylation substrate of Sirt6.

Next, we sought to identify the deacetylation sites of XBP1s. HEK293T cells were transfected with p300 with or without Sirt6, and XBP1s immunoprecipitates were analyzed by LC-MS/MS. Compared to cells transfected with p300 acetyltransferase only, four lysine residues (K65, K146, K257, and K297) were identified as deacetylated in cells overexpressing Sirt6 (Figs. 5c, S6a, Table S1). To determine whether the half-life of the XBP1s protein is increased when deacetylation at these residues is prevented by the presence of Sirt6, we substituted each residue with arginine (K65R, K146R, K257R, and K297R). We expressed either WT or mutated XBP1s along with Sirt6 and then repeated the CHX chase experiment. The stability of XBP1s was significantly increased with K257R and K297R but not with K65R and K146R compared to that of WT XBP1s (Figs. 5d and S6c, d), indicating that Sirt6 deacetylation of K257 and K297 mediates XBP1s protein degradation. Next, to assess whether acetylation of XBP1s plays a role in hepatic lipid accumulation, we injected C57BL/6 mice with adenoviruses carrying either XBP1-WT or double-mutant XBP1s (2KR, K257/297R). As shown in Fig. 5e, hepatic TG levels in mice with AdXBP1s-WT, but not with AdXBP1s-2KR, were higher compared to those with AdLacZ. Increases in steatosis induced by XBP1s-WT were further confirmed by perilipin immunostaining (Fig. S7a). Furthermore, XBP1s-WT, but not XBP1s-2KR, induced hepatic apoptosis and insulin resistance in mice and hepatocytes, respectively (Fig. S7b, S7c). These results collectively indicate that XBP1s acetylation at Lys257 and Lys297 is critical in XBP1s-mediated hepatic steatosis, apoptosis, and insulin resistance. 


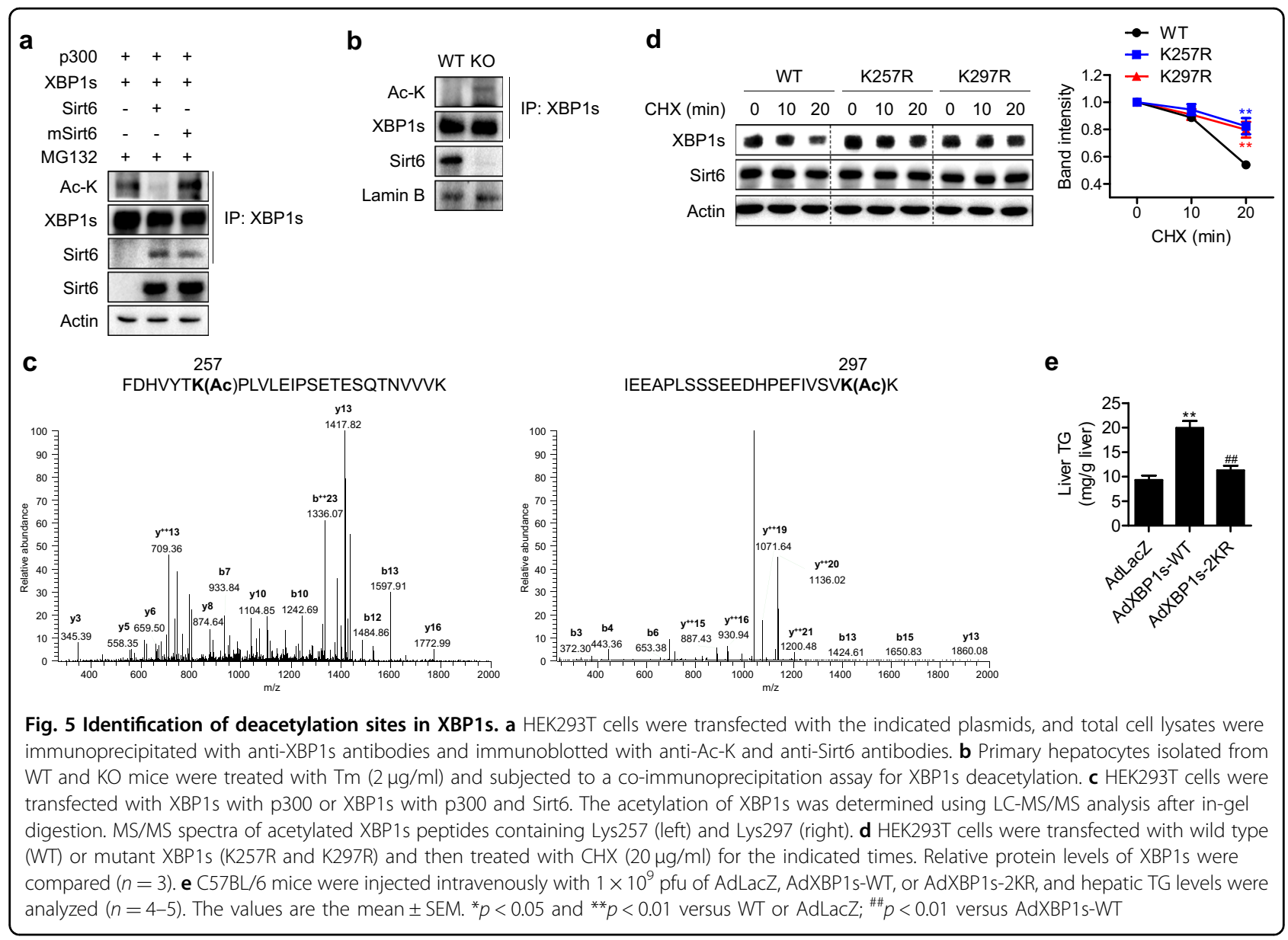

Pharmacological activation of Sirt6 attenuates Tm-induced ER stress and fat accumulation in the liver

Recently, Rahnasto-Rilla et $\mathrm{al}^{29}$. identified that fucoidan, which is a natural sulfated polysaccharide extracted from brown seaweed, is a Sirt6 activator. Therefore, we aimed to evaluate the effects of fucoidan on ER stressmediated hepatic steatosis. WT and Sirt6 KO mice were administered fucoidan via oral gavage three times, as depicted in Fig. 6a, and liver tissue was analyzed. We first confirmed that fucoidan acted as a Sirt6 activator in mice by finding decreased H3K9 acetylation in WT livers, which was not observed in KO livers (Fig. 6b). Fucoidan administration effectively suppressed Tm-induced XBP1s and CHOP expression and TG accumulation in the livers of WT mice but not in the livers of Sirt6 KO mice (Fig. 6b-d), indicating that alleviation of the ER stress response by fucoidan was mediated by Sirt6. The results obtained from further experiments in vitro, in which HepG2 cells were treated with fucoidan or a structurally distinct Sirt6 activator cyanidin ${ }^{30}$, were in good agreement with the in vivo data in that treatment with these agents decreased the levels of total and acetylated XBP1s in a concentration-dependent manner
(Fig. 6e, f). Again, successful activation of Sirt6 by fucoidan and cyanidin was indicated by a reduction in Ac-H3K9.

\section{The XBP1s signaling pathway is activated in HFD- and HFHF-fed Sirt6 KO mice}

To determine whether the XBP1s pathway is altered by nutrient status, we analyzed hepatic XBP1s and CHOP expression in various NAFLD animal models with low Sirt6 expression. Compared to control mice, dietary (i.e., HFD, MCD, or HFHF) or genetic ( $d b / d b)$ mice models of NAFLD had markedly elevated levels of both total and acetylated XBP1s and CHOP protein in liver (Fig. 7a). The impact of Sirt6 deficiency was further investigated in mice fed a HFD or HFHF to physiologically mimic NAFLD development in humans. When fed a HFD, hepatic steatosis was enhanced in $\mathrm{KO}$ mice, as identified by hepatic TG levels (Fig. 7b) and immunostaining for hepatic lipid droplets (Fig. 7c). Similar to the results of the Tm study, the XBP1s pathway was upregulated even more in the livers of HFD- and HFHF-fed Sirt6 KO mice than in the livers of WT mice (Fig. 7d, e). 


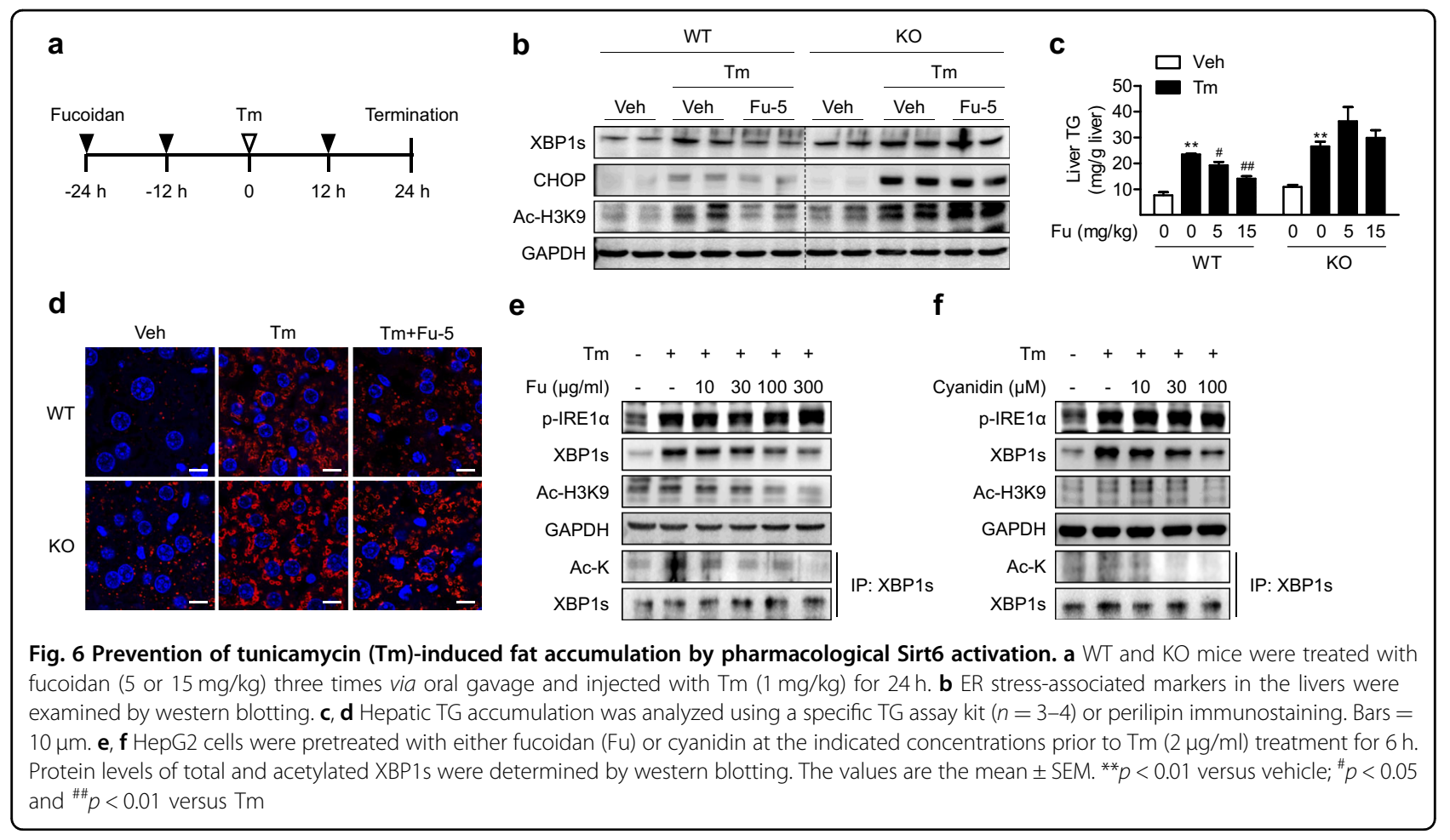

a

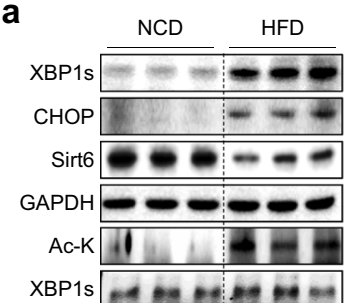

C

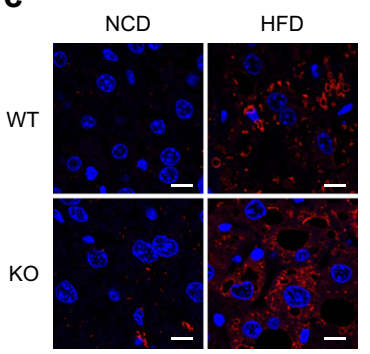

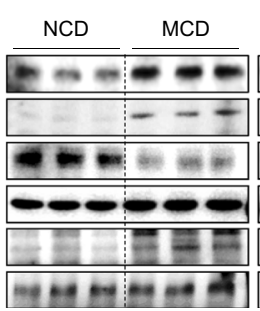

d
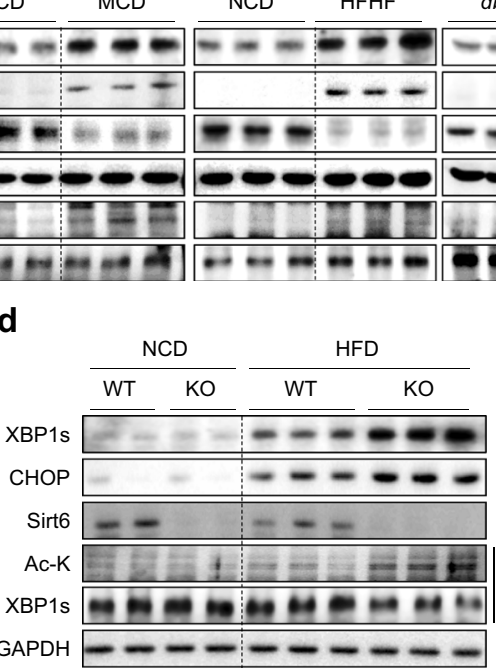

b

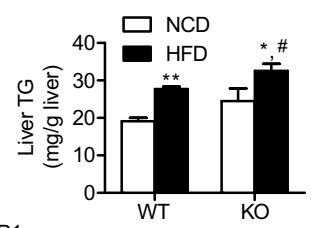

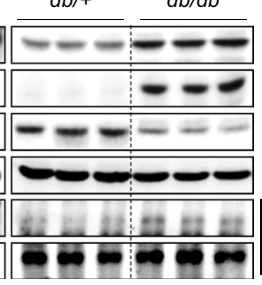

\section{IP: XBP1s}

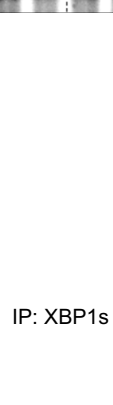

e

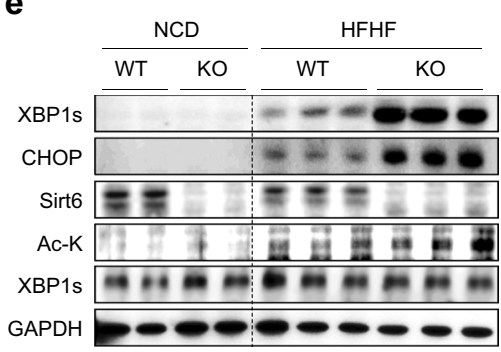

Fig. 7 Activation of the XBP1s pathway in diet-induced animal models of NAFLD by Sirt6 deficiency. a Protein levels of XBP1s and CHOP were analyzed in 10-week HFD-fed mice, 4-week MCD-fed mice, and 10-week HFHF-fed mice and in 12-week-old db/db mice. b, c Hepatic TG levels and perilipin immunostaining for lipid droplets were determined. Bars $=10 \mu \mathrm{m}$. d, e Protein levels of XBP1s and CHOP were compared in NCD-, HFD-, or HFHF-fed WT and KO mice. The values are the mean \pm SEM. ${ }^{*} p<0.05$ and ${ }^{* *} p<0.01$ versus NCD; $p<0.05$ versus WT

XBP1 acetylation is increased in patients with NAFLD

To provide clinical relevance, we compared the XBP1s acetylation status in patients with NAFLD and healthy subjects. Total and acetylated XBP1s levels were noticeably higher in the liver tissues of NAFLD patients than in those of healthy controls (Fig. 8a). Consistent with our previous report ${ }^{24}$, Sirt6 protein levels were decreased in NAFLD patients. The most important finding was that the NAFLD activity score (NAS) was highly positively correlated with the levels of acetylated XBP1s but negatively correlated with Sirt6 expression (Fig. 8b). Moreover, AcXBP1s was negatively correlated with Sirt6 expression, 

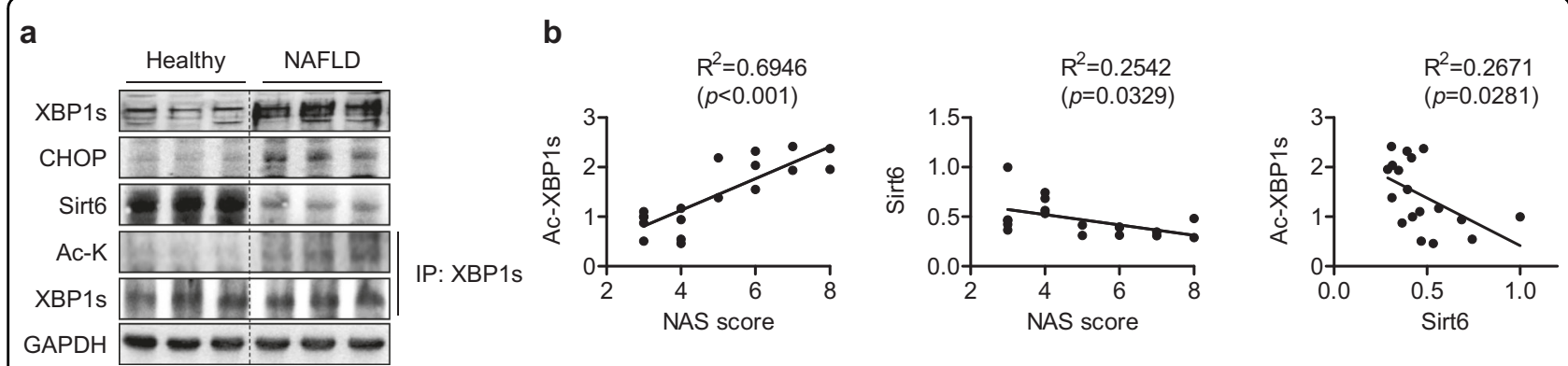

Fig. 8 Increased XBP1s acetylation in the liver of NAFLD patients. a Expression of total and acetylated XBP1s and Sirt6 in livers from healthy subjects and NAFLD patients was analyzed by western blotting. b The correlation between NAFLD activity score (NAS), Ac-XBP1s and Sirt6 ( $n=18)$

indicating that Sirt6 directly mediates XBP1s deacetylation. These findings suggest that the reduced expression of Sirt6 and concordant increases in acetylation, and thus $\mathrm{XBP} 1 \mathrm{~s}$ expression, contribute to the disease progression of NAFLD in patients.

\section{Discussion}

This study was designed to address the hypothesis that Sirt6 ameliorates hepatic ER stress by deacetylating XBP1s to improve fat accumulation, apoptosis, and insulin resistance in hepatocytes. The plausibility of this hypothesis was derived from the previous finding that prolonged and unmitigated ER stress is tightly linked to NAFLD $^{7,31}$ and that hepatic insulin resistance and steatosis in NAFLD are aggravated by Sirt6 deficiency ${ }^{24,25}$. Here, we showed for the first time that hepatocyte-specific Sirt6 deficiency exacerbated the XBP1s pathway of the UPR, at least in part, by regulating the protein stability of XBP1s via its deacetylation (Fig. S8). Additionally, we demonstrated that Sirt6 overexpression or pharmacological activation counteracted Tm-induced ER stress to alleviate hepatic steatosis and associated pathologies.

In this study, several lines of evidence suggest that Sirt6 has a protective role against ER stress. First, Sirt6 KO mice exhibited an aggravated ER stress response compared with WT mice after Tm administration or highcalorie diet feeding. Among the three pathways of UPR signaling, activation of XBP1s, and to a lesser extent the PERK pathway, but not ATF6, had a significant impact on the ER stress response in Sirt6 KO hepatocytes. Supporting this notion, the protein levels of XBP1s and its target genes CHOP, EDEM, and ERDJ4 were greatly increased in the livers of $\mathrm{KO}$ mice. In contrast, genetic overexpression and pharmacological activation of Sirt6 in hepatocytes were sufficient to reduce the activation of the XBP1s pathway, both in vitro and in vivo.

Second, Tm-stimulated hepatic steatosis was increased in Sirt6 KO mice but decreased by Sirt6 augmentation, which was assessed by perilipin immunostaining and the TG content of the liver. Because a variety of molecules in the liver are supposed to mediate the dysregulation of lipid metabolism under ER stress, we sought to determine which genes or pathways were involved in the attenuation of steatosis by Sirt6. Unlike previous reports, lipogenic gene (e.g., SREBP-1c, fatty acid synthase, stearoyl-CoA desaturase, and diacylglycerol acetyltransferase 2) and very low-density lipoprotein receptor expression levels were not increased by Tm stimulation. Furthermore, the Tm-induced change in the expression of PPAR $\alpha$, which is essential for fatty acid oxidation, was modest. The expression of all these genes was preserved upon Sirt6 KO or overexpression. These results imply that ER stress inducers, or the resulting adaptive response, regulate hepatic lipid metabolism in a way that cannot be simply attributed to a single metabolic pathway.

Third, Sirt6 deficiency resulted in impaired insulin signaling and increased apoptosis under ER stress, whereas Sirt6 overexpression and Sirt6 activation improved hepatic insulin resistance and apoptosis. Previous studies have shown that reducing ER stress with chemical chaperones improves insulin resistance and cell injury, $7,32,33$. It is likely that all three UPR signaling pathways are fundamental to ER stress-induced apoptosis. Among others, CHOP, whose induction depends on XBP1s, ATF4, and ATF6, is known to promote apoptotic cell death partly by blocking the anti-apoptotic effect of $\mathrm{Bcl}^{34}$. In the current study, Sirt6 strongly inhibited $\mathrm{XBP} 1 \mathrm{~s}$ activation and, to a lesser extent, PERK phosphorylation; both of these effects might contribute to marked CHOP inhibition and subsequent hepatocyte apoptosis. In addition, ER stress induces hepatic insulin resistance mainly through lipotoxicity and XBP1s. The inhibition of XBP1s activation by siRNA has been found to protect against palmitate-induced downregulation of Akt phosphorylation ${ }^{35}$. Thus, diminished XBP1s activation by Sirt6 might contribute to the restoration of hepatic insulin sensitivity in our study.

Finally, total and acetylated XBP1s protein levels were significantly higher in the liver tissues of NAFLD patients than in those of healthy controls, suggesting an inhibitory 
role of Sirt6-mediated deacetylation of XBP1s in NAFLD pathogenesis. We found here that Sirt6 deficiency led to strong activation of the XBP1s pathway under ER stress. Because the production of XBP1s is largely dependent on XBP1 mRNA splicing, which is regulated by IRE1 $\alpha^{36}$, we assessed the levels of p-IRE1 $\alpha$ as well as XBP1u and XBP1s mRNA and found that there were no differences between the genotypes. Combining these data with the finding that deacetylase-inactive mSirt6 had no effect on $\mathrm{XBP} 1 \mathrm{~s}$ levels, we hypothesized that the increase in XBP1s in Sirt6 KO mice may be mediated by post-translational modification, i.e., deacetylation. Although the acetylation/ deacetylation status has been shown to significantly affect the stability and activity of the XBP1s protein ${ }^{9,15,32}$, the acetylation of which specific lysine residues plays a critical role remains unknown. Through LC-MS/MS analysis and a subsequent functional study, we identified that Sirt6 deacetylates XBP1s at Lys65, Lys146, Lys257, and Lys297 and that the deacetylation of the last two residues leads to its degradation through a proteasome-mediated pathway. Our results indicate a reduction in Sirt6 expression in NAFLD and other conditions associated with ER stress, and the consequent increase in XBP1s activity via acetylation-dependent protein stabilization leads to enhanced steatosis, insulin resistance, and injury in the liver. Indeed, in human liver, the expression of acetylated $\mathrm{XBP} 1 \mathrm{~s}$ is highly correlated with NAS in NAFLD patients with reduced Sirt6 expression.

In light of the fact that acetyl-CoA is abundant as an intermediary product of glucose catabolism, histone acetylation has emerged as a link between epigenetic regulation and NAFLD development and has thus gained increasing attention over the past decade as a therapeutic option against NAFLD. For instance, hepatocyte-specific HDAC3 deletion caused steatosis ${ }^{37,38}$, and this was due to enhanced hepatic de novo lipogenesis after release from protein complexes with transcription repressors. Although our current study focused on the deacetylation of a nonhistone protein, i.e., XBP1s, by Sirt6, all of these studies support the notion that (de)acetylation of histone or nonhistone substrates may provide an important targeting strategy against hepatic fat accumulation and associated pathologies. Altogether, Sirt6 activation may play a protective role against ER stress-induced NAFLD progression.

\section{Acknowledgements \\ This work was supported by grants from the Medical Research Center Program (NRF-2017R1A5A2015061) and by grants from the Basic Science Research Program (2017R1A2B4008593 and 2019R1A2C2002281) of the National Research Foundation (NRF), which is funded by the Korean government (MSIP).}

\section{Author details}

'Department of Biochemistry, Chonbuk National University Medical School, Jeonju, Jeonbuk 54896, Republic of Korea. ${ }^{2}$ College of Pharmacy, Kyungpook
National University, Daegu 41566, Republic of Korea. ${ }^{3}$ Department of Pathology, Chonbuk National University Medical School, Jeonju, Jeonbuk 54896, Republic of Korea. ${ }^{4}$ Department of Physiology, Gachon University College of Medicine, Incheon 21999, Republic of Korea. ${ }^{5}$ College of Pharmacy,

Chonbuk National University, Jeonju, Jeonbuk 54896, Republic of Korea

\section{Author contributions}

I.H.B., O.K.K., L.H., D.P., M.J.C., B.C.O., and S.L. performed the experiments and analyzed the data. E.J.B. and B.H.P. designed the experiments, interpreted the data, and wrote the manuscript. All authors reviewed the manuscript.

\section{Conflict of interest}

The authors declare that they have no conflict of interest.

\section{Publisher's note}

Springer Nature remains neutral with regard to jurisdictional claims in published maps and institutional affiliations.

Supplementary information accompanies this paper at https://doi.org/ 10.1038/s12276-019-0309-0.

Received: 21 February 2019 Revised: 29 May 2019 Accepted: 7 June 2019. Published online: 20 September 2019

\section{References}

1. Angulo, P. Nonalcoholic fatty liver disease. N. Engl. J. Med. 346, 1221-1231 (2002).

2. Malhi, H. \& Kaufman, R. J. Endoplasmic reticulum stress in liver disease. J. Hepatol. 54, 795-809 (2011).

3. Walter, P. \& Ron, D. The unfolded protein response: from stress pathway to homeostatic regulation. Science 334, 1081-1086 (2011).

4. Yoshida, H., Matsui, T., Yamamoto, A., Okada, T. \& Mori, K. XBP1 mRNA is induced by ATF6 and spliced by IRE1 in response to ER stress to produce a highly active transcription factor. Cell 107, 881-891 (2001).

5. Pagliassotti, M. J. Endoplasmic reticulum stress in nonalcoholic fatty liver disease. Annu Rev. Nutr. 32, 17-33 (2012).

6. Noureddin, M., Mato, J. M. \& Lu, S. C. Nonalcoholic fatty liver disease: update on pathogenesis, diagnosis, treatment and the role of S-adenosylmethionine. Exp. Biol. Med. 240, 809-820 (2015).

7. Ozcan, U. et al. Endoplasmic reticulum stress links obesity, insulin action, and type 2 diabetes. Science 306, 457-461 (2004).

8. Jo, H. et al. Endoplasmic reticulum stress induces hepatic steatosis via increased expression of the hepatic very low-density lipoprotein receptor. Hepatology 57, 1366-1377 (2013).

9. Wang, S. et al. IRE1alpha-XBP1s induces PDI expression to increase MTP activity for hepatic VLDL assembly and lipid homeostasis. Cell Metab. 16, 473-486 (2012).

10. Zhang, K. et al. The unfolded protein response transducer IRE1alpha prevents ER stress-induced hepatic steatosis. EMBO J. 30, 1357-1375 (2011).

11. Jurczak, M. J. et al. Dissociation of inositol-requiring enzyme (IRE1alpha)mediated c-Jun N-terminal kinase activation from hepatic insulin resistance in conditional X-box-binding protein-1 (XBP1) knock-out mice. J. Biol. Chem. 287, 2558-2567 (2012).

12. Lee, A. H., Scapa, E. F., Cohen, D. E. \& Glimcher, L. H. Regulation of hepatic lipogenesis by the transcription factor XBP1. Science 320, 1492-1496 (2008).

13. Cao, J. et al. Endotoxemia-mediated activation of acetyltransferase P300 impairs insulin signaling in obesity. Nat. Commun. 8, 131 (2017).

14. Pan, Q. R. et al. Resveratrol prevents hepatic steatosis and endoplasmic reticulum stress and regulates the expression of genes involved in lipid metabolism, insulin resistance, and inflammation in rats. Nutr. Res. 35, 576-584 (2015).

15. Wang, F. M., Chen, Y. J. \& Ouyang, H. J. Regulation of unfolded protein response modulator XBP1s by acetylation and deacetylation. Biochem J. 433, 245-252 (2011).

16. Shin, J. et al. SIRT7 represses Myc activity to suppress ER stress and prevent fatty liver disease. Cell Rep. 5, 654-665 (2013).

17. Michishita, E. et al. SIRT6 is a histone H3 lysine 9 deacetylase that modulates telomeric chromatin. Nature 452, 492-496 (2008). 
18. Michishita, E. et al. Cell cycle-dependent deacetylation of telomeric histone $\mathrm{H3}$ lysine K56 by human SIRT6. Cell Cycle 8, 2664-2666 (2009).

19. Tasselli, L. et al. SIRT6 deacetylates H3K18ac at pericentric chromatin to prevent mitotic errors and cellular senescence. Nat. Struct. Mol. Biol. 23, 434-440 (2016).

20. Dominy, J. E. et al. The deacetylase Sirt6 activates the acetyltransferase GCN5 and suppresses hepatic gluconeogenesis. Mol. Cell 48, 900-913 (2012).

21. Jang, H. Y., Gu, S., Lee, S. M. \& Park, B. H. Overexpression of sirtuin 6 suppresses allergic airway inflammation through deacetylation of GATA3. J. Allergy Clin. Immunol. 138, 1452-1455 (2016).

22. Moon, Y. J. et al. Sirtuin 6 in preosteoclasts suppresses age- and estrogen deficiency-related bone loss by stabilizing estrogen receptor a. Cell Death Differ. (2019). https://doi.org/10.1038/s41418-019-0306-9.

23. Woo, S. J. et al. Myeloid sirtuin 6 deficiency accelerates experimental rheumatoid arthritis by enhancing macrophage activation and infiltration into synovium. EBioMedicine 38, 228-237 (2018).

24. Ka, S. O., Bang, I. H., Bae, E. J. \& Park, B. H. Hepatocyte-specific sirtuin 6 deletion predisposes to nonalcoholic steatohepatitis by up-regulation of Bach1, an Nrf2 repressor. FASEB J. 31, 3999-4010 (2017).

25. Kim, H. S. et al. Hepatic-specific disruption of SIRT6 in mice results in fatty liver formation due to enhanced glycolysis and triglyceride synthesis. Cell Metab. 12, 224-236 (2010).

26. Kleiner, D. E. et al. Design and validation of a histological scoring system for nonalcoholic fatty liver disease. Hepatology 41, 1313-1321 (2005).

27. Wang, J. et al. Sirtuin 2 aggravates postischemic liver injury by deacetylating mitogen-activated protein kinase phosphatase-1. Hepatology 65, 225-236 (2017).

28. Qiang, L. et al. Brown remodeling of white adipose tissue by SirT1-dependent deacetylation of Ppargamma. Cell 150, 620-632 (2012).

29. Rahnasto-Rilla, M. K. et al. The identification of a SIRT6 activator from brown algae Fucus distichus. Mar. Drugs 15, E190 (2017).

30. Rahnasto-Rilla, M. et al. Natural polyphenols as sirtuin 6 modulators. Sci. Rep. 8 4163 (2018)
31. Kammoun, H. L. et al. GRP78 expression inhibits insulin and ER stress-induced SREBP-1C activation and reduces hepatic steatosis in mice. J. Clin. Invest. 119 1201-1215 (2009).

32. Uemura, A. et al. UBC9 regulates the stability of XBP1, a key transcription factor controlling the ER stress response. Cell Struct. Funct. 38, 67-79 (2013).

33. Park, S. M., Choi, J., Nam, T. G., Ku, J. M. \& Jeong, K. Anti-diabetic effect of 3hydroxy-2-naphthoic acid, an endoplasmic reticulum stress-reducing chemical chaperone. Eur. J. Pharm. 779, 157-167 (2016).

34. Szegezdi, E., Logue, S. E., Gorman, A. M. \& Samali, A. Mediators of endoplasmic reticulum stress-induced apoptosis. EMBO Rep. 7, 880-885 (2006).

35. Achard, C. S. \& Laybutt, D. R. Lipid-induced endoplasmic reticulum stress in liver cells results in two distinct outcomes: adaptation with enhanced insulin signaling or insulin resistance. Endocrinology 153, 2164-2177 (2012).

36. Tirosh, B., Iwakoshi, N. N., Glimcher, L. H. \& Ploegh, H. L. Rapid turnover of unspliced Xbp-1 as a factor that modulates the unfolded protein response. J. Biol. Chem. 281, 5852-5860 (2006).

37. Feng, D. et al. A circadian rhythm orchestrated by histone deacetylase 3 controls hepatic lipid metabolism. Science 331, 1315-1319 (2011).

38. Sun, Z. et al. Hepatic Hdac3 promotes gluconeogenesis by repressing lipid synthesis and sequestration. Nat. Med. 18, 934-942 (2012).

39. Lee, H. S. et al. Overexpression of sirtuin 6 suppresses inflammatory responses and bone destruction in mice with collagen-induced arthritis. Arthritis Rheum. 65, 1776-1785 (2013).

40. Luo, J. et al. A protocol for rapid generation of recombinant adenoviruses using the AdEasy system. Nat. Protoc. 2, 1236-1247 (2007).

41. Shevchenko, A., Tomas, H., Havlis, J., Olsen, J. V. \& Mann, M. In-gel digestion for mass spectrometric characterization of proteins and proteomes. Nat. Protoc. 1, 2856-2860 (2006)

42. Chen, Y., Kwon, S. W., Kim, S. C. \& Zhao, Y. Integrated approach for manual evaluation of peptides identified by searching protein sequence databases with tandem mass spectra. J. Proteome Res. 4, 998-1005 (2005). 